\title{
Impact of Adherence Education and Monitoring on Community Pharmacy Performance Scores and Patient Satisfaction
}

Chelsea Sprouse, PharmD*1; Morgan Grant, PharmD ${ }^{1}$; Jamie Remines, PharmD ${ }^{1}$; Pramit Nadpara, PhD²; Jean-Venable Goode, PharmD, BCPS, FAPhA, FCCP

${ }^{1}$ Kroger Health; ${ }^{2}$ Virginia Commonwealth University

*At time of writing, PGY1 Community-Based Pharmacy Resident, Virginia Commonwealth University

\section{ABSTRACT}

Background: Adherence, specifically to noninsulin diabetes medications, statins, and renin-angiotensin system antagonists (i.e. angiotensin-converting-enzyme inhibitors (ACEi), angiotensin II receptor blockers (ARBs), and aliskiren), is a measure tracked by the Centers for Medicare and Medicaid Services (CMS) to give Medicare Part D plans a star rating; pharmacies are impacted by these star ratings. The pharmacy is given a performance score based on the measure. Some pharmacies use a performance information management platform (PIMP) that allows pharmacies to better understand performance information to impact patient care.

Objectives: (1) To evaluate if a monthly adherence monitoring and education service impacts the percentage of patients adherent determined by pharmacy performance scores; (2) To determine patient satisfaction with the service in a large community pharmacy chain.

Methods: A six-month prospective interventional pilot study including patients with a proportion of days covered (PDC) of less than $80 \%$ for oral diabetes or renin-angiotensin system antagonists (RASA) medications was conducted in two pharmacies of a large community pharmacy chain in Southwest Virginia. Using pharmacy internal data analytics and PIMP data, the percentage of patients who are adherent to oral diabetes or RASA medications was determined including the baseline PDC for each patient. At the start of the study, the standard of care in this large community chain pharmacy was to address adherence, follow-up in one month and every three months thereafter. In this study, pharmacists provided monthly telephonic adherence monitoring and education for a six-month period. Each session was scripted for medication adherence history, education and data collection. The pharmacist provided guidance and counseling based on how the patient answered the questions. Pharmacists gathered information about adherence patterns and behaviors using a 14 item Likert-scale and multiple choice-based questionnaire during the first session. After completion of the final adherence monitoring and education session, pharmacists gathered patient satisfaction information using an eight item Likert-scale questionnaire. At the end of the six-month period, using PIMP data, the percentage of patients adherent to oral diabetes or RASA medications was determined based on pharmacy performance scores. The data was analyzed using uni-variate and bi-variate statistics to determine if there was a difference in pharmacy performance scores from the pre-study analysis. The adherence patterns and behaviors, as well as patient satisfaction with the program was evaluated to determine factors influencing nonadherence.

Results: A total of 55 patients were identified in two pharmacies, ten of which were excluded or declined involvement, leaving 45 enrolled in the adherence monitoring and education service. Of the 45 enrolled patients, ten completed the adherence patterns and behaviors questionnaire. About half of the patients were men (50.95\%) with an average age of 71.17 years and taking an average of 6.55 prescription medications. All patients had Medicare Part D insurance and majority had a yearly income of less than $\$ 40,000$. The average baseline PDC was 68.92. In pharmacy 1, the average performance score for oral diabetes medications trended down and the average performance score for hypertension medications trended up over the study period. In pharmacy 2, the average performance score for oral diabetes medications trended up and the average performance score in hypertension medications trended up over the study period. The adherence patterns and behaviors questionnaire revealed the majority of patients rarely forgot to take medications or run out of medications. Additionally, cost of medications did not seem to impact adherence and majority of patients knew the names and indications of their medications. Only one patient completed the patient satisfaction survey.

Conclusions: An adherence monitoring and education service had mixed results in improving patient adherence and pharmacy performance scores. Only one patient completed the satisfaction survey, thus no conclusions can be made regarding patient satisfaction of the program. More research needs to be done regarding telephonic adherence programs.

Keywords: patient education, medication adherence, community pharmacy

Corresponding author:

Jean-Venable Goode, PharmD, BCPS, FAPhA, FCCP

Virginia Commonwealth University

Email: jrgoode@vcu.edu

\section{BACKGROUND}

The World Health Organization (WHO) defines adherence as being "the extent to which a person's behavior - taking medication, following a diet, and or executing lifestyle changes - corresponds with the agreed recommendations from a health care provider." ${ }^{1}$ This definition highlights the importance of communication and involvement of a health care professional with the patient to manage their health. It is estimated that 
between $20-50 \%$ of patients are nonadherent to their prescribed regimens. ${ }^{2}$ Adherence can be affected by the relationship that the patient has with the health care provider or health care system. ${ }^{3}$ Evidence suggests that the more chronic disease states a person has, as well as the number of medications a person is taking can significantly affect adherence. ${ }^{3}$ This problem is more prominent in the elderly population, where $40-86 \%$ of patients are nonadherent. In the United States, $33-69 \%$ of all medication-related hospital admissions are due to poor medication adherence. ${ }^{2}$ Nonadherence to medications results in a higher number of patients who do not achieve the benefits of the medication and as a consequence, they experience poor health outcomes and poor quality of life, which ultimately leads to increased health care costs. $^{3}$

Previous studies suggest that intensive care with patient counseling, education, and training programs, provided by a pharmacist, can improve medication adherence. ${ }^{4-7,13,14}$ Murray and colleagues led a study in which a pharmacist provided a 9month multi-level adherence counseling intervention, with a 3month post-study phase and concluded that medication adherence was $67.9 \%$ and $78.8 \%$ in the usual care and intervention groups, respectively. Also, in the 3-month postintervention follow-up period, adherence was $66.7 \%$ and $70.6 \%$, respectively. ${ }^{4}$ Lee and colleagues tested the efficacy of a pharmacy care program to improve medication adherence and determined that after six months of intervention, the average medication adherence increased from $61.2 \%$ to $96.9 \% .^{5}$ A study done by Pringle measured the change in proportion of days covered (PDC) in 5 chronic medication classes over a 12-month period after interventions by a pharmacist and concluded that in the intervention group, PDC improved in all five chronic medication classes. ${ }^{6}$ Another study aimed to assess the effectiveness of a medication adherence intervention and determine the overall impact of the intervention on adherence rates and Centers for Medicare and Medicaid Services (CMS) Part D "star rating" adherence measures. The researchers concluded that patient adherence rates increased for all drug classes studied and star ratings for the health plan were also significantly improved. ${ }^{7}$

Adherence is often measured and tracked using a PDC calculation, which is based on the fill dates and day supply for each fill of a prescription. ${ }^{8}$ Adherence, specifically to noninsulin diabetes, renin angiotensin system antagonists (RASA) (i.e. angiotensin-converting-enzyme inhibitors (ACEi), angiotensin II receptor blockers (ARBs), and aliskiren), and statin medications, is one of the measures that CMS tracks in order to give Medicare Part D plans a "star rating". The CMS "star rating" system uses a range from one to five stars to denote how a Medicare Part D plan is performing overall or with regards to a specific measure. The system helps to provide beneficiaries with information about the quality of available Medicare Part D plans. For example, five stars would mean excellent performance and one star would mean poor performance. ${ }^{9}$
CMS does not offer star ratings to pharmacies, only to insurance plans. However, insurance plans can track and assess how pharmacies are meeting quality measures that impact performance. Thus, insurance plans may give their own ratings to pharmacies based on the success of certain quality measures; these ratings are often referred to as performance scores. Based on the ratings the pharmacy achieves, insurance plans can determine whether or not they want a certain pharmacy to be included in their network. There are many factors that determine ratings and performance scores, one of which is medication adherence. Pharmacists should be engaged in services including medication management to achieve high performance scores, which will in turn allow the pharmacy to be on the preferred network list with third-party health plans. ${ }^{10}$

In order to track performance, some pharmacies use a performance information management platform (PIMP) that makes unbiased, benchmarked performance data available to both health plans and community pharmacy organizations. The PIMP uses prescription and medical data, calculates performance and displays a score every month. These platforms partner with insurance payers directly to streamline performance measurements; all performance scores, from various payers, are used to create one standard set of measurements. ${ }^{11}$

\section{PHARMACY ADHERENCE MONITORING SERVICE}

The large community pharmacy chain has an adherence monitoring service already in place for patients who meet certain criteria. With the help of a PIMP, corporate analytics identifies patients who are taking noninsulin oral diabetes, RASA, and/or statin medications who also have a low PDC $<$ $80 \%$ ) for these medications. These patients are considered "high-priority", meaning that they have a specific prescription insurance plan that offers the pharmacy ratings in adherence measures; these patients directly affect the pharmacy's performance scores. The pharmacy adherence performance scores are a population score that these "high priority" patients impact negatively with low adherence. The population scores were used for the research (an average of all scores coming from third-party insurance plans).

The corporate pharmacy team places an adherence intervention for these patients into a clinical queue system for pharmacists to access and improve care at the store level. Once a patient is identified as nonadherent, a pharmacist makes an intervention with a patient with the intent to help improve medication adherence. The nonadherence standard of care is to provide the patient with adherence monitoring when he/she first populates into the clinical queue. Adherence monitoring is provided to the patient after one month and then quarterly thereafter; via telephone or in person. Pharmacists do not have a script to follow during these encounters and often, pharmacists provide a varying level of counseling and education to the patient. However, the corporation is always making 
adjustments in the adherence monitoring program to better serve patients at the pharmacy level.

This research study aims to improve the current standard of care in patient adherence education. The purpose of this study is to evaluate if a monthly adherence monitoring and education service impacts the percentage of patients adherent determined by pharmacy performance scores and to determine patient satisfaction with the service in two pharmacies of a large community pharmacy chain.

\section{METHODS}

This prospective interventional pilot study was conducted in two pharmacies of a large community pharmacy chain stores in Southwest Virginia. Prior to the start of the study, investigator Sprouse trained the pharmacists on the study protocol and how to provide counseling and education using pre-existing standard counseling guidelines, which were used during each monthly session (Appendix A). The pharmacists were also trained on how to appropriately use the adherence monitoring and education scripts (Appendices B-D), which were developed by the investigators.
Baseline data was collected in December 2018 including demographics (age and gender) and the PDC for each patient was recorded and the enrollment period occurred from January 1, 2019 to March 31, 2019. The final patient was enrolled in March 2019 and was tracked for six months through September 2019.

At the start of the study, using the PIMP data and internal corporate analytics, the percentage of patients adherent to oral diabetes and RASA medications was determined by adherence measure performance scores at each of the two pharmacies. Nonadherent high-priority patients were automatically populated into the pharmacy clinical queue as part of the pharmacy's standard workflow. Patients who met the inclusion criteria (Table 1) were called to ask if he/she wanted to enroll in the study and be monitored for six months. The pharmacist obtained consent for telephone interviews during the initial phone call. For this study, pharmacists at each store provided patients with adherence monitoring and education on a monthly basis, mostly via telephone, for six months. Figure 1 describes study enrollment and methods.

Table 1. Inclusion and exclusion criteria

\begin{tabular}{|c|l|}
\hline Inclusion & Exclusion \\
\hline $\begin{array}{l}\text { 18 years or older } \\
\text { Patients pre-identified by the PIMP and pharmacy } \\
\text { internal analytics }\end{array}$ & $\bullet \quad \begin{array}{l}\text { Patients who are lost to follow-up } \\
\text { Incomplete/missing documentation of an } \\
\text { encounter }\end{array}$ \\
$\begin{array}{l}\text { Patient taking oral diabetes medications and/or } \\
\text { RASA medications (ACE-I, ARB, direct renin } \\
\text { inhibitor) }\end{array}$ & $\bullet \quad \begin{array}{l}\text { Prisoners, children } \\
\end{array}$ \\
\hline
\end{tabular}




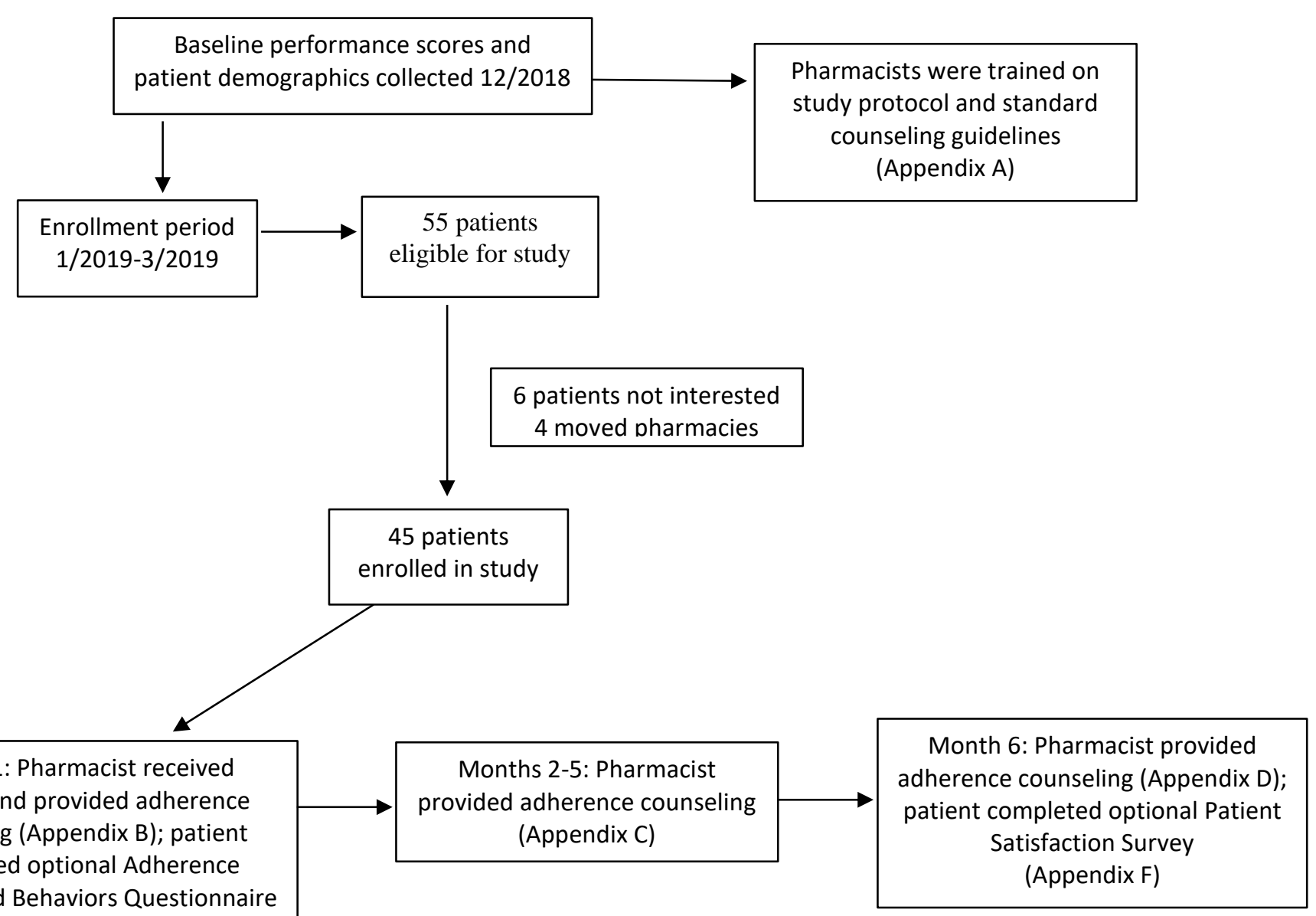

(Appendix E)

Figure 1. Flow chart of enrollment and adherence counseling methods.

During the initial adherence monitoring and education session (month 1), there was a script (Appendix B) that contained medication adherence questions, as well as education to be provided to the patient. The pharmacist provided guidance and counseling based on how the patient answered the questions. Also during the initial session, the patient completed an optional Adherence Patterns and Behaviors Questionnaire, which was developed by investigators based on previous literature. For the following sessions (months 2-5), the pharmacist used Appendix $C$ for a script and for the last session (month 6), the pharmacist used Appendix D for a script. However, each session used the same set of counseling guidelines. Also during the final session, patients completed an optional Patient Satisfaction Survey (Appendix F).

For patients who were not reachable by telephone, a hard-stop was placed in the pharmacy dispensing system to alert the pharmacist on duty to complete the appropriate counseling session. When the patient presented to the pharmacy, the education and adherence session was provided face to face. All 45 patients enrolled in the study were contacted each month via telephone or in person at the pharmacy; no patients were lost to follow-up. Additionally, for each counseling session the pharmacist on duty documented in the patient's electronic chart how much time was spent during the encounter. The study information was available in the pharmacy at all times.

Each month, using PIMP data and corporate analytics, the percentage of patients adherent to oral diabetes and RASA medications was determined by adherence measure performance scores at each pharmacy; descriptive statistics were used to depict adherence rates and performance measures. The primary outcome of this study was to determine if there was a change in percentage of patient adherent in oral diabetes and RASA medications, determined by adherence measure performance scores. The secondary outcome was to determine patient satisfaction of the adherence monitoring and education service. 
At the end of the study, the Adherence Patterns and Behaviors Questionnaire was analyzed using descriptive statistics to determine the biggest influences on nonadherence. The average time for each encounter with each patient was calculated from all the encounters documented in each patient's electronic file. The Patient Satisfaction Survey was analyzed using descriptive statistics to determine how satisfied patients were with the medication adherence monitoring and education service provided by pharmacists.

\section{RESULTS}

Between two pharmacies, 55 patients were identified as eligible for the study. Six patients were not interested in the study and four patients moved to another pharmacy. Forty-five patients were enrolled and received monthly follow-up. Patient demographics (Table 2) were collected in December 2018, prior to the first adherence monitoring and education session.

Table 2. Patient demographics and baseline pharmacy performance scores.

\begin{tabular}{|c|c|}
\hline \multicolumn{2}{|c|}{ Patient Demographics ( $\mathbf{n}=\mathbf{4 5}$ ) and Pharmacy Performance Scores } \\
\hline Male (\%) & 50.95 \\
\hline Age (average years, (range)) & $71.17(47-101)$ \\
\hline Average number of prescription medications & 6.55 \\
\hline Primary Prescription Insurance Plan (\%) & Medicare Part D (100) \\
\hline PDC at baseline (average \%, (range)) & $96.92(50.63-79.47)$ \\
\hline Baseline average performance score in diabetes, \\
pharmacy 1 \\
\hline Baseline average performance score in diabetes, \\
pharmacy 2
\end{tabular}

Of the 45 patients enrolled, ten patients completed the optional Adherence Patterns and Behaviors Questionnaire (Figures 2 and 3). Majority of patients (71\%) indicated an annual income of less than $\$ 40,000$ and $67 \%$ indicated they never take less medication due to high cost. Majority of patients (73\%) indicate they never or rarely forget to take their medications. Patients can mention the names of their medications and can mention what their medications are for, $60 \%$ and $50 \%$ respectively.

\section{Adherence Patterns and Behaviors Questionnaire Part 1 $(n=10)^{*}$}

I take my medications every day at a fixed time or on fixed occasions

How often do you take less medication because of high cost?

How often do you forget to get prescriptions filled at the pharmacy?

How does your health now limit you in moderate activities, such as moving a table, bowling, playing golf, or other activities?

How often do you miss taking your medications because you feel better or healthier?

How often do you run out of medication?

How often do you forget to take your medications?
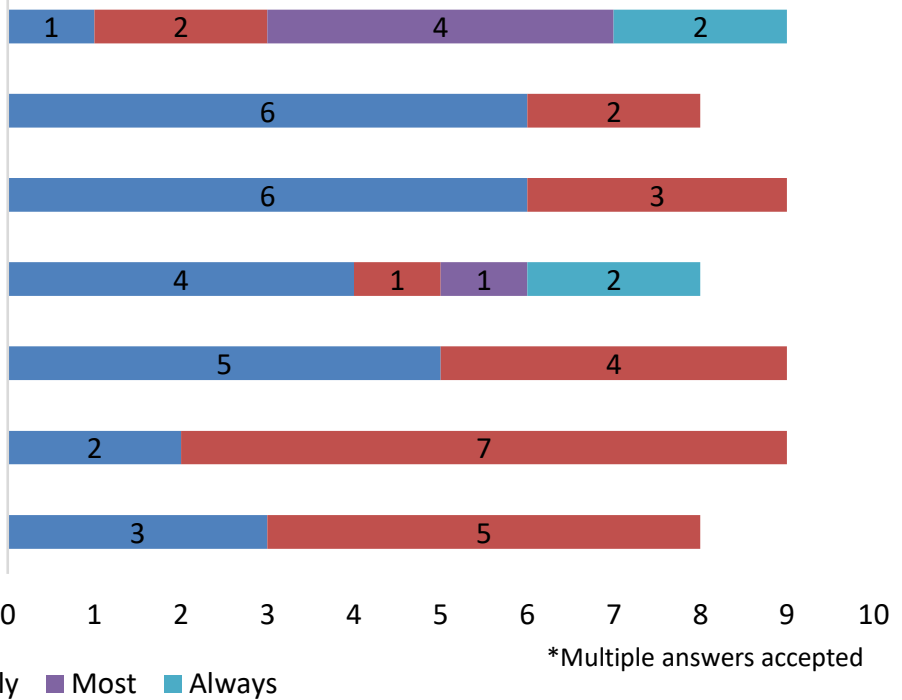

None $\quad$ Rarely $\square$ Most $\quad$ Always

Figure 2. Adherence Patterns and Behaviors Questionnaire.

Figure contains the answers to the questions in the first-half of the questionnaire. 


\section{Adherence Patterns and Behaviors Questionnaire Part 2 $(n=10)^{*}$}

I am really afraid of the side effects of my medications

I feel basically healthy. Therefore, I am sometimes unsure whether I really have to take my medications daily

I can mention what my medications are for, without hesitation

I can mention the names of my medications, without hesitation

\begin{tabular}{|c|c|c|c|c|}
\hline & & 0 & 2 & 6 \\
\hline Strongly Disagree & Disagree & Neutral & Agree & Strongly Agree \\
\hline
\end{tabular}
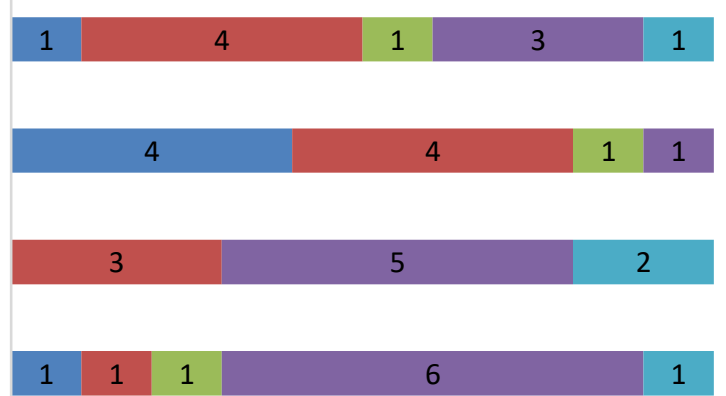

810

12

*Multiple answers accepted

Figure 3. Adherence Patterns and Behaviors Questionnaire.

Figure contains the answers to the questions in the second-half of the questionnaire.

Evaluation of pharmacy 1 from the start of the study to the end of the study, the percentage of patients adherent to oral diabetes medications trended down and the average pharmacy score also trended down (Figure 4). Evaluation of pharmacy 2 revealed the percentage of patients adherent to oral diabetes medications trended down however, the average pharmacy score trended up (Figure 5). In pharmacy 1, the percentage of patients adherent to RASA medications remained steady, while the average pharmacy score trended up (Figure 6). In pharmacy 2, the percentage of patients adherent to RASA medications trended down slightly however, the average pharmacy score trended up (Figure 7).

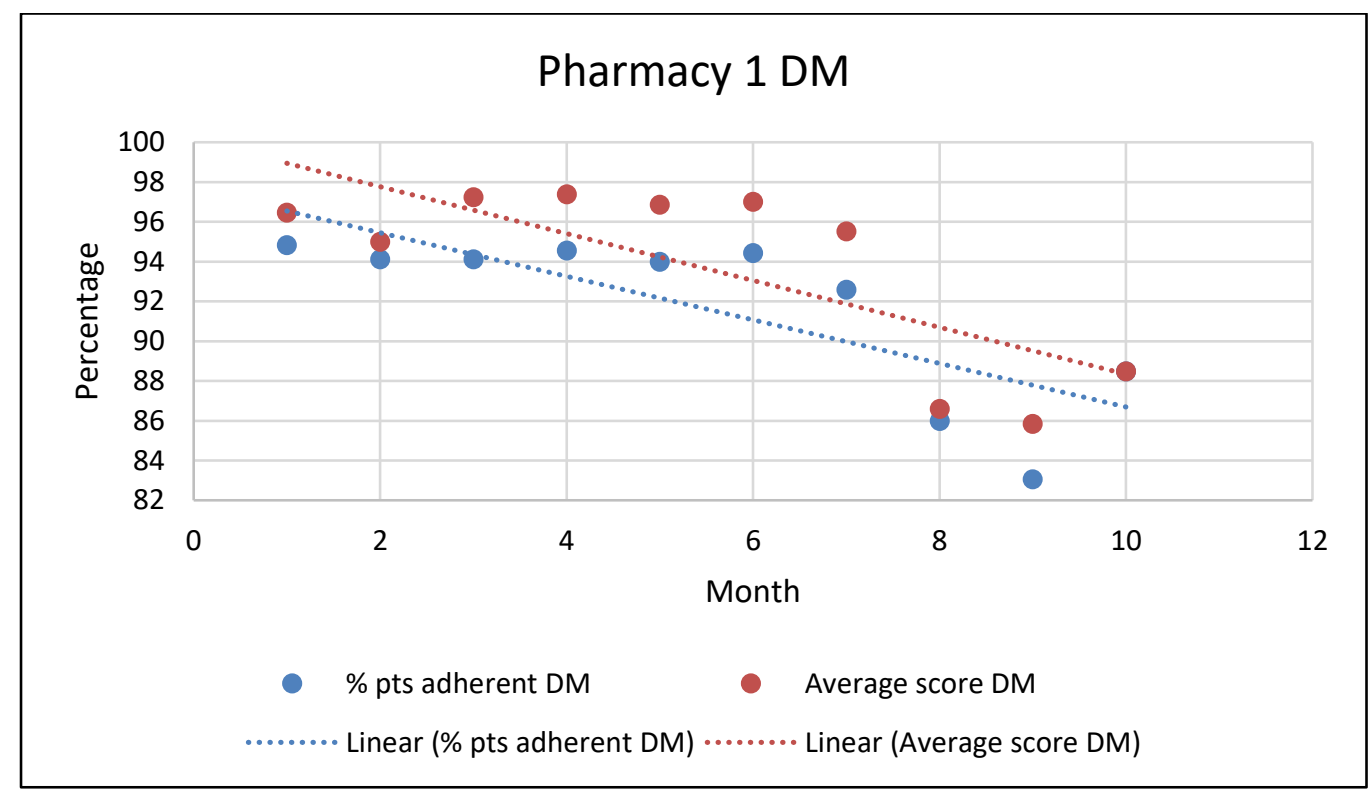

Figure 4. In pharmacy 1, the percentage of patients adherent in diabetes medications and the average pharmacy score are shown over the study period (month 1 being December and month 10 being September). 


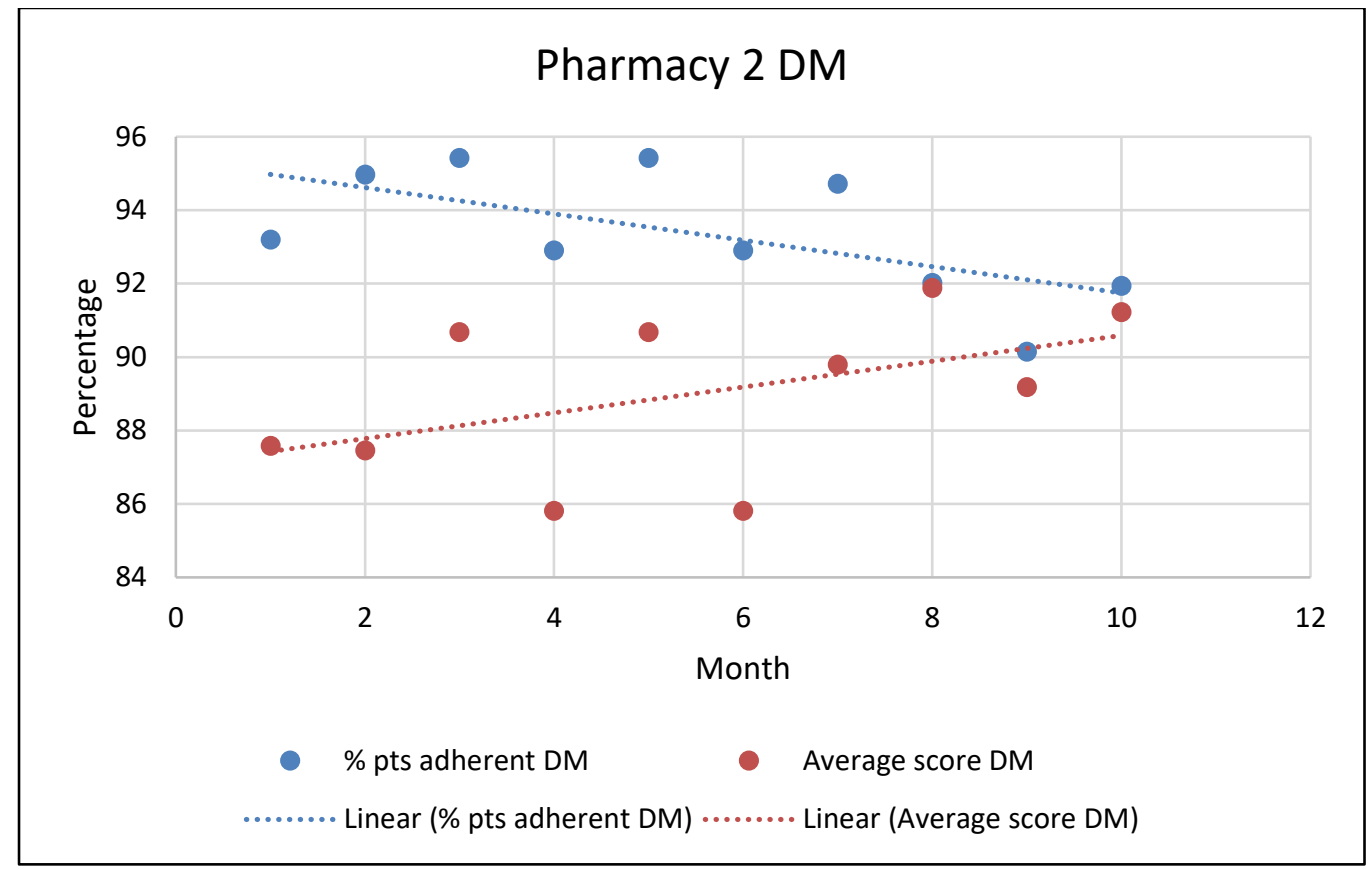

Figure 5. In pharmacy 2, the percentage of patients adherent in diabetes medications and the average pharmacy score are shown over the study period (month 1 being December and month 10 being September).

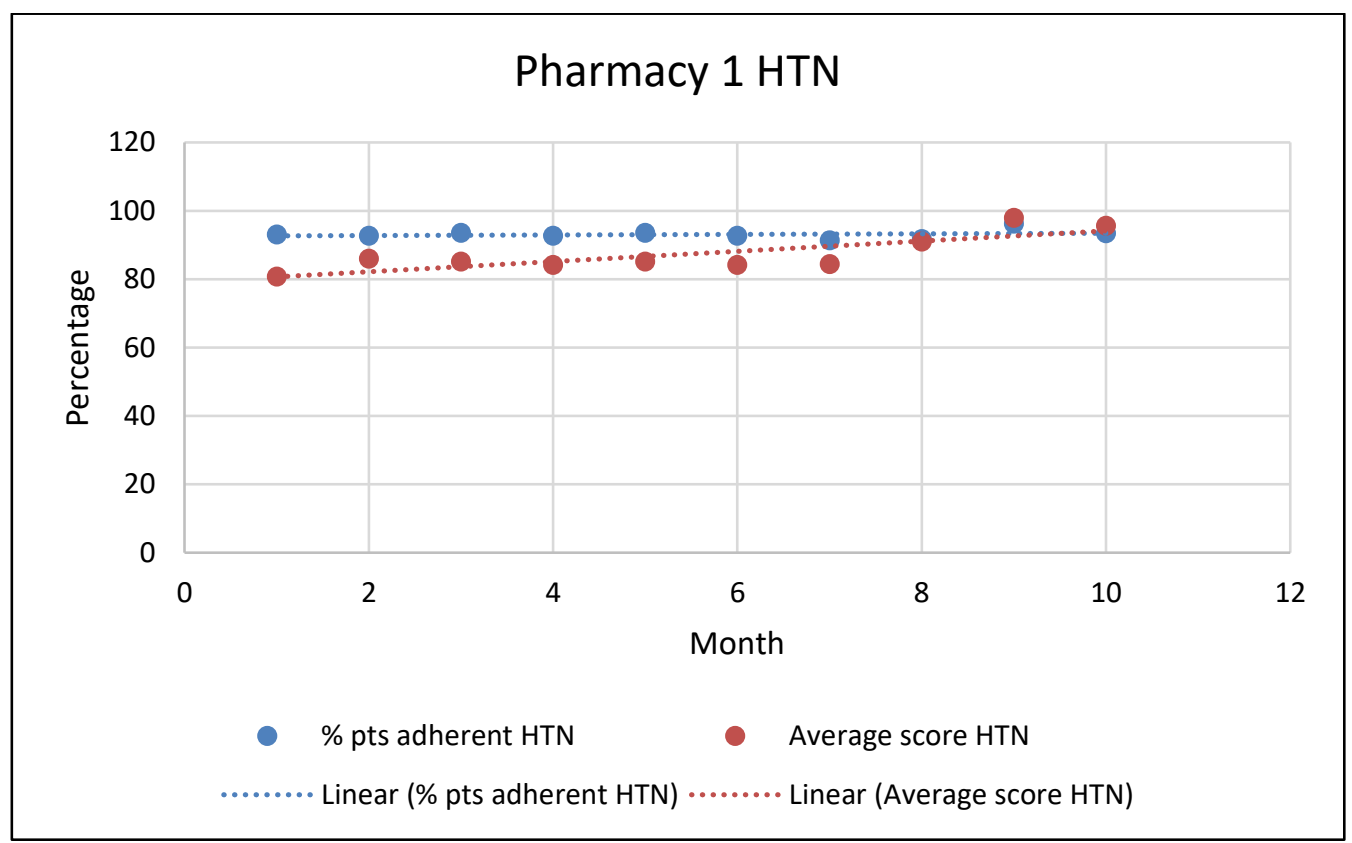

Figure 6. In pharmacy 1, the percentage of patients adherent in hypertension medications and the average pharmacy score are shown over the study period (month 1 being December and month 10 being September). 


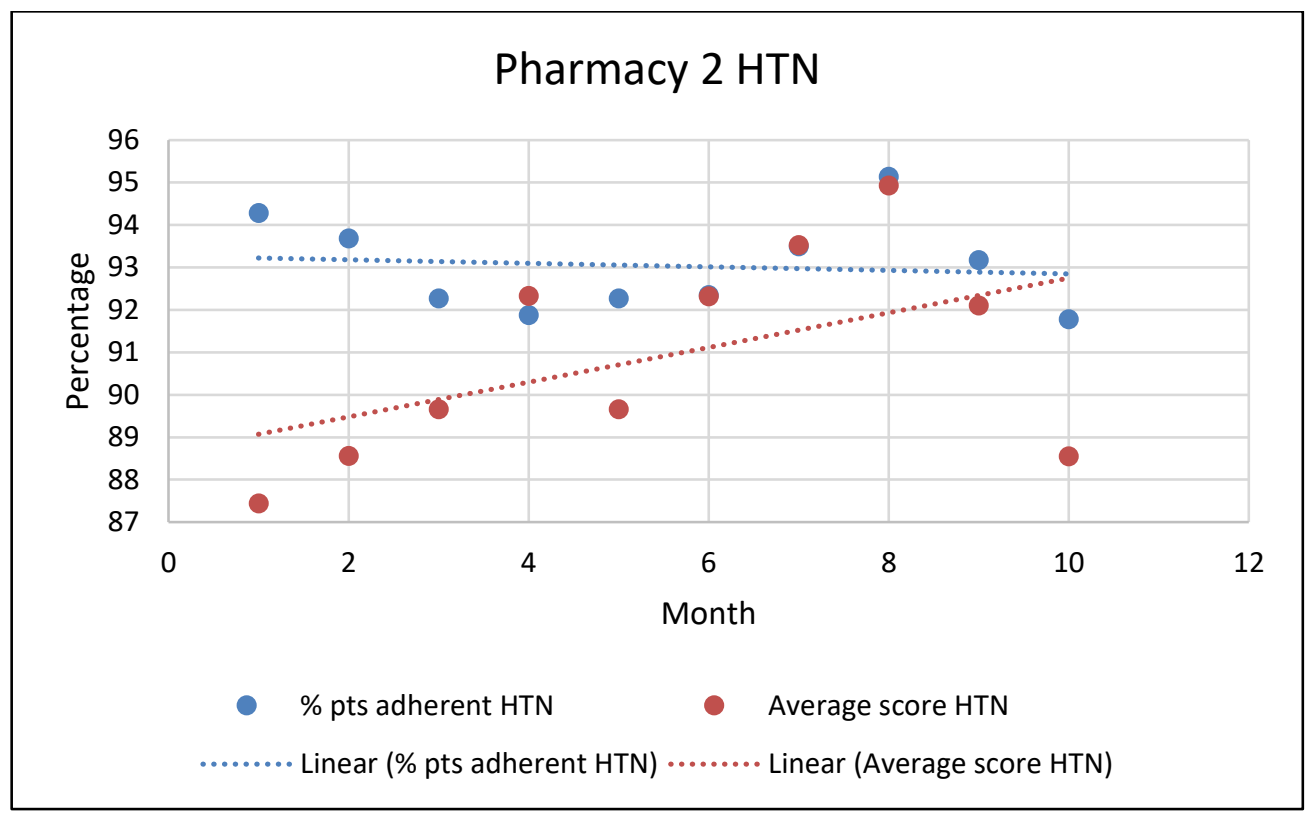

Figure 7. In pharmacy 2, the percentage of patients adherent in hypertension medications and the average pharmacy score are shown over the study period (month 1 being December and month 10 being September).

The average time for the initial interview between both pharmacies was 9.27 minutes (range 5-15 minutes). The average time for subsequent interviews was 6.95 minutes (range 4-10 minutes). The majority of patient interactions each month were in person at the pharmacy because patients did not answer the phone calls from the pharmacy. At the end of the study, one patient completed the optional patient satisfaction survey.

\section{DISCUSSION}

The results of this study show that engaging patients in an adherence monitoring and education service may or may not improve patient adherence and lead to increased pharmacy performance scores. A previous pharmacist-led telephone intervention study resulted in an increased PDC after a sixmonth period, following an initial interview. The study targeted patients with comorbid hypertension and diabetes. Even though the study showed an increase in adherence following one telephone call, the researchers suggest that it may be more beneficial to have follow-up calls. ${ }^{2}$

A study protocol was published in The Netherlands that looked at the effects of a telephone counseling intervention 2 or 3 weeks following a new prescription; the study assessed medication adherence, patient beliefs and patient satisfaction. The results were not published, but the study mentions that unintentional non-adherence can be a result of personal beliefs regarding medications, chronic disease and socio- demographics. ${ }^{14}$ Similarly, our study looked at patient demographics and used a survey to gain knowledge about our patients' behaviors so that the pharmacist could tailor the counseling interventions for a specific patient. It is important to gauge a patient's beliefs and behaviors about taking medications so the pharmacist can identify barriers to adherence and have a meaningful intervention. ${ }^{14}$ Different from the previously mentioned studies, our study took a step further and aimed to improve patient adherence after multiple follow-up calls.

In our study, majority of patients indicated an annual income of less than $\$ 40,000$ however, majority indicated they never take less medication because of high cost. Majority of patients also indicate they never or rarely forget to take their medications, leading to the conclusion that many patients believe to be adherent even though they may not be. This leads back to the idea that it is important to identify unintentional nonadherence before attempting to make meaningful interventions.

Over the six-month study period, the pharmacy performance scores varied each month. Ideally, improvement in patient adherence would lead to improved pharmacy performance scores, however this is not always the case. This is because many third-party insurance plans are giving performance scores to the pharmacy and often performance scores are reported as an average. The average pharmacy performance score is 
dependent upon the total number of adherent patients in their respective insurance plans. However, outliers in the total number of adherent patients within a specific insurance plan can drastically affect the performance, despite having a higher average percentage of patients adherent. For example, if comparing two scenarios the percentage of patients adherent can stay the same but the average pharmacy performance score can be significantly influenced. Furthermore, an increase (or decrease) in pharmacy performance scores does not always mean there was an increase (or decrease) in patients adherent. This is one explanation of the study results in pharmacy 2 (Figure 5), how the percentage of patients adherent to oral diabetes medications trended down, but the average pharmacy performance score trended up.

The initial interviews were on average longer because of the explanation of the study process and consent needed from the patient, in addition to the counseling and optional survey. The subsequent interviews were on average shorter. Since the majority of the interviews were between five and seven minutes, this type of adherence monitoring service is feasible in the community pharmacy setting.

More research with a larger cohort needs to be done to see if monthly adherence check-ins improve patient adherence. Patients may have been more willing to participate if there were incentives in place. The baseline performance score for each pharmacy was greater than 80; this was a limitation because even though the scores may have increased, there was little room for drastic improvement. Another limitation was that even though each pharmacist was trained prior to the start of the study, there could have been some variability in the education provided by different pharmacists. There were no direct incentives for the pharmacists providing counseling. However, one could argue that the incentive would be that the pharmacy could become a preferred pharmacy for more insurance plans if the performance scores increased. One could also argue that higher pharmacy performance scores may lead to salary raises or bonuses for individual pharmacy personnel.

Furthermore, this study was not designed to capture patients that changed insurance plans throughout the year. If a patient originally had a plan that did not offer pharmacy performance scores, then changed to a plan that did offer pharmacy performance scores, the study did not account for that and would not have enrolled this patient in the study. Or vice-versa, if a patient originally had a plan that did offer pharmacy performance scores, then changed to a plan that did not offer pharmacy performance scores, the investigators would have continued to follow this patient even though his/her adherence did not affect the pharmacy's performance score. This may be a reason why in pharmacy 2 we saw a decrease in patients adherent to RASA medications, but still saw an increase in pharmacy performance scores. Therefore, further studies need to be done that follow "high-priority" patients only, accounting for those who changed insurance plans throughout the year.
Other than lack of interest in being a part of a research study, several patients declined enrollment because the research was mostly telephone-based and they did not want more phone calls from the pharmacy. Contact with patients by telephone was a significant barrier for this research study. This leads to the question of whether or not telephone-based services are an impactful or beneficial means of communication to provide patient care. Previous research suggests that telephone-based services are convenient for patients and are associated with better adherence outcomes versus conventional methods. ${ }^{12}$ With conflicting evidence, more research needs to be done regarding telephone-based pharmacy services. Additionally, our study did not specifically address patient satisfaction with telephone-based encounters, therefore further studies need to be conducted to assess patient satisfaction with these services.

\section{CONCLUSIONS}

An adherence monitoring and education service had mixed results in improving patient adherence and pharmacy performance scores. Since only one patient completed the patient satisfaction survey, no conclusions can be made to determine patient satisfaction with this service. More research needs to be done to find the most effective means of communication to patients regarding improvement in medication adherence.

Poster presentation: American Pharmacists Association Annual Meeting; Seattle, WA; March 2019

Podium presentation: University of North Carolina Research in Education and Practice Symposium; Chapel Hill, NC; May 2019

Conflicts of Interest: We declare no conflicts of interest or financial interests that the authors or members of their immediate families have in any product or service discussed in the manuscript, including grants (pending or received), employment, gifts, stock holdings or options, honoraria, consultancies, expert testimony, patents and royalties.

Treatment of Human Subjects: IRB review/approval required and obtained 


\section{REFERENCES}

1. Sabaté, E. Adherence to Long-Term Therapies. World Health Organization 2003.

https://www.who.int/chp/knowledge/publications/a dherence_full_report.pdf?ua=1. Accessed 7/25/18.

2. Abughosh SM et al. A Pharmacist Telephone Intervention to Identify Adherence Barriers and Improve Adherence Among Nonadherent Patients with Comorbid Hypertension and Diabetes in a Medicare Advantage Plan. JMCP 2016; 22(1):63-73.

3. Costa $\mathrm{E}$ et al. Interventional tools to improve medication adherence: review of literature. Patient Prefer Adherence 2015; 9:1303-1314.

4. Murray MD et al. Pharmacist Intervention to Improve Medication Adherence in Heart Failure: A Randomized Trial. Anns Intern Med 2007; 146(10):714-725.

5. Lee JK et al. Effect of a Pharmacy Care Program on Medication Adherence and Persistence, Blood Pressure, and Low-Density Lipoprotein Cholesterol. JAMA 2006; 296(21):2563-2571.

6. Pringle JL et al. The Pennsylvania Project: Pharmacist Intervention Improved Medication Adherence and Reduced Health Care Costs. HealthAffairs 2014. Retrieved from https://doi.org/10.1377/hlthaff.2013.1398. Accessed 7/25/18.

7. Leslie RS et al. Evaluation of an Integrated Adherence Program Aimed to Increase Medicare Part D Star Rating Measures. JMCP 2014; 20(12):1193-1203.

8. Nau DP. Proportion of Days Covered (PDC) as a Preferred Method of Measuring Medication Adherence. Pharmacy Quality Alliance. Retrieved from http://www.pqaalliance.org/images/uploads/files/PQ A\%20PDC\%20vs\%20\%20MPR.pdf. Accessed 7/31/18.
9. Brandt NJ and Cooke CE. Centers for Medicare and Medicaid Services Support for Medication Therapy Management (Enhanced Medication Therapy Management) Testing Strategies for Improving Medication Use Among Beneficiaries Enrolled in Medicare Part D. Clin Geriatr Med 2017; 33:153-164.

10. American Pharmacies (2018). How CMS Star Ratings Will Affect Your Revenue. Retrieved from http://www.aprx.org/issues-advocacy/star-ratingsoverview. Accessed 7/28/18.

11. Pharmacy Quality Solutions Inc. EQuIPP ${ }^{\text {тM }} 2019$. Retrieved from: https://www.pharmacyquality.com/equippplatform/. Accessed 4/17/19.

12. Zullig LL et al. Improving diabetes medication adherence: successful, scalable interventions. Patient Prefer Adherence 2015; 9:139-149.

13. Zanni GR. Counseling for Improved Medication Adherence. Pharmacy Times 2011. Retrieved from https://www.pharmacytimes.com/publications/issue /2011/september2011/counseling-for-improvedmedication-adherence. Accessed 7/25/18.

14. Kooy MJ et al. Effects of a TELephone Counseling Intervention by Pharmacist (TelCIP) on medication adherence, patient beliefs and satisfaction with information for patients starting treatment: study protocol for a cluster randomized controlled trial. BMC Health Serv Res 2014; 14(219). 


\section{APPENDIX A}

\section{Forms of Medication Nonadherence ${ }^{13}$}

- Failing to initially fill or refill prescriptions

- Omitting medications or taking more than the prescribed doses

- Prematurely discontinuing medications

- Taking doses at the wrong time

- Taking medications with prohibited foods, liquids, or other medications

- Taking outdated or damaged medications

- Improperly storing medications

\section{Standardized Counseling Guidelines for Pharmacist ${ }^{13}$}

- Avoid asking yes/no questions that may elicit a false-negative response (i.e. "Are you taking your medication?") Begin by asking, "How many times have you missed taking your medication in the past week/month?"

- Probe for reasons behind skipped doses

- Communicate clearly and avoid technical jargon

- Because poor health literacy is linked to nonadherence, ask patients, "What is this medication is for?" If the patient answers incorrectly, review the agent, including the proper way to take it.

- $\quad$ Probe for side effects. Reducing side-effect profile improves adherence. When possible, recommend an alternative with fewer side effects.

- If possible, simplify the medication regimen. Switching to once-daily dosing results in twice as many adherent days as more complex dosing

- Forgetfulness is a major factor in nonadherence. Recommend memory aids (alarms, email reminder, notes on refrigerators, placement of medication in a prominent location, pill organizers, calendars).

- Express confidence that the patient can do better; use positive reinforcement. If possible, involve other family members or caregivers.

- Review of time lags for medications, meaning that it could take days/weeks before patient notices a difference when beginning a new medication.

- Emphasize proper dosing because skipping a dose is common. Provide guidelines for when doses are skipped. Emphasize the impact of not taking medications properly.

- If cost is impacting adherence, recommend pharmacy assistance programs/coupons/etc. 


\section{APPENDIX B}

Script for INITIAL adherence monitoring and education session (MONTH 1)

"Hello, my name is [INSERT NAME] and I am a pharmacist here at Kroger. Do you have a few minutes to talk with me? [PATIENT RESPONSE] I am doing a project that looks to improve Kroger's contact with our patients and I would like to contact you on a monthly basis to see how you are doing with your medications. Is this something that you would be willing to take part in? [PATIENT RESPONSE] Before we begin, I must review some consent information with you. This information is meant to assist you in carefully thinking about whether being in this study is right for you and your situation. Please ask me to explain anything that is not clear to you. You are invited to participate in a research study about improving patient adherence. If you agree to participate, I will ask you some questions about your medications. This interview will take approximately ten minutes and approximately 125 people will participate. Your participation is voluntary. You may choose to not participate in this interview, stop the interview at any time, or skip any questions. Information that you give me will be kept as confidential as possible by storing it in secure databases accessible only to the following people: study personnel, authorized people at VCU or VCUHS who oversee research, and authorized officials of the Department of Health and Human Services. The information collected as part of this study will not be used or distributed for future research studies, even if identifiers are removed. As part of this research study, we will ask you to share identifiable health information with us and/or permit us to access existing information from your healthcare records. This type of information is considered "Protected Health Information" that is protected by federal law. To conduct this research, we may use your health and medication record. By agreeing to this study, you authorize VCU and VCU Health to use and/or share your health information for this research. The health information just described may be used by and/or shared with the following people and groups to conduct, monitor, and oversee the research: the Principal Investigator and Research Staff, Research Collaborators, Data Coordinators, Institutional Review Boards, and Others as Required by Law. Once your health information has been disclosed to anyone outside of this study, the information may no longer be protected under this authorization. This authorization will expire when the research study is closed, or there is no need to review, analyze and consider the data generated by the research project, whichever is later. You may change your mind and take back the right to use your protected health information at any time. However, even if you take back your Authorization, the researchers may still use or release any health information that they have already collected about you for the study. If you take back this Authorization, you may no longer be allowed to participate in the research study. To take back this Authorization, you must write to:

Chelsea Sprouse

72 Kingston Dr.

Daleville, VA 24083

If you have any questions, concerns, or complaints about your participation in this research, please contact Kelly Goode at 804-8283000. If you want to talk to someone separate from the research team, please contact the VCU Office of Research at 804-827-2157.

Do you have any questions about this study? [PATIENT RESPONSE]

Do you consent to participate in this research interview? [PATIENT RESPONSE]

Ok, great! I see that you take [INSERT ORAL DIABETES MEDICATION OR RASA MEDICATION]. What do you take this medication for? [PATIENT RESPONSE] How do you take this medication? [PATIENT RESPONSE] What did the doctor tell you to expect from this medication? [PATIENT RESPONSE] How many times per week do you miss a dose of this medication? [PATIENT RESPONSE] What is the reason for your missed doses, if applicable? [PATIENT RESPONSE] What methods do you use to remember to take your medications (pill box, phone alarm, calendars, etc.)? [PATIENT RESPONSE].

- Counseling on oral diabetic medications: It is important to take your medication as prescribed by your doctor because diabetes can lead to many complications if not managed correctly and effectively. Some of the complications include worsened eye sight and even blindness, decreased sensation in your legs and feet, which could lead to amputations, as well as kidney failure, stroke, and heart attack

- Counseling on RASA medications: It is important to take your medication as prescribed by your doctor because high blood pressure is considered a "silent killer" and blood pressure lowering medications are one of the best influences for high blood pressure. These medications can help control blood pressure and can reduce your risk of heart attack and stroke.

Thank you for allowing me to explain the benefits of taking your medication as prescribed. I am participating in a research study and would appreciate it if you would complete a brief survey. The survey is voluntary. Your survey will be kept in a secure location in the pharmacy and will be confidential. ["ADHERENCE PATTERNS AND BEHAVIORS QUESTIONNAIRE”]. Thank you for your time and please let me know if you have any questions." 


\section{APPENDIX C}

Script for monthly adherence monitoring and education sessions (MONTHS 2-5)

"Hello, my name is [INSERT NAME] and I am a pharmacist here at Kroger. Do you have a few minutes to talk with me about your medications? [PATIENT RESPONSE] I am looking to improve Kroger's contact with our patients and I would like to contact you on a monthly basis to see how you are doing. I see that you take [INSERT ORAL DIABETES MEDICATION OR RASA MEDICATION]. What do you take this medication for? [PATIENT RESPONSE] How do you take this medication? [PATIENT RESPONSE] What did the doctor tell you to expect from this medication? [PATIENT RESPONSE] How many times per week do you miss a dose of this medication? [PATIENT RESPONSE] What is the reason for your missed doses, if applicable? [PATIENT RESPONSE] What methods do you use to remember to take your medications (pill box, phone alarm, calendars, etc.)? [PATIENT RESPONSE].

- Counseling on oral diabetic medications: It is important to take your medication as prescribed by your doctor because diabetes can lead to many complications if not managed correctly and effectively. Some of the complications include worsened eye sight and even blindness, decreased sensation in your legs and feet, which could lead to amputations, as well as kidney failure, stroke, and heart attack

- Counseling on RASA medications: It is important to take your medication as prescribed by your doctor because high blood pressure is considered a "silent killer" and blood pressure lowering medications are one of the best influences for high blood pressure. These medications can help control blood pressure and can reduce your risk of heart attack and stroke.

Thank you for allowing me to explain the benefits of taking your medication as prescribed and please let me know if you have any questions."

\section{APPENDIX D}

Script for LAST monthly adherence monitoring and education session (MONTH 6)

"Hello, my name is [INSERT NAME] and I am a pharmacist here at Kroger. Do you have a few minutes to talk with me about your medications? [PATIENT RESPONSE] I am looking to improve Kroger's contact with our patients and I would like to contact you on a monthly basis to see how you are doing. I see that you take [INSERT ORAL DIABETES MEDICATION OR RASA MEDICATION]. What do you take this medication for? [PATIENT RESPONSE] How do you take this medication? [PATIENT RESPONSE] What did the doctor tell you to expect from this medication? [PATIENT RESPONSE] How many times per week do you miss a dose of this medication? [PATIENT RESPONSE] What is the reason for your missed doses, if applicable? [PATIENT RESPONSE] What methods do you use to remember to take your medications (pill box, phone alarm, calendars, etc.)? [PATIENT RESPONSE].

- Counseling on oral diabetic medications: It is important to take your medication as prescribed by your doctor because diabetes can lead to many complications if not managed correctly and effectively. Some of the complications include worsened eye sight and even blindness, decreased sensation in your legs and feet, which could lead to amputations, as well as kidney failure, stroke, and heart attack

- Counseling on RASA medications: It is important to take your medication as prescribed by your doctor because high blood pressure is considered a "silent killer" and blood pressure lowering medications are one of the best influences for high blood pressure. These medications can help control blood pressure and can reduce your risk of heart attack and stroke.

Thank you for allowing me to explain the benefits of taking your medication as prescribed. I am participating in a research study and would appreciate it if you would complete a brief survey. The survey is voluntary. Your survey will be kept in a secure location in the pharmacy and will be confidential. ["PATIENT SATISFACTION SURVEY"]. Thank you for your time and please let me know if you have any questions." 


\section{APPENDIX E}

Adherence Patterns and Behaviors Questionnaire

1. How many medications (prescription and over-the-counter) do you take on a regular basis:

2. Please select annual income (select one option):
A. $<\$ 10,000$
B. $\$ 10,000-40,000$
C. $\$ 40,000-80,000$
D. $>\$ 80,000$

3. How do you pay for the majority of your medications (select one option):
A. Commercial insurance
B. Medicare D
C. Medicaid
D. Cash/no insurance

\begin{tabular}{|c|c|c|c|c|c|}
\hline & None & Rarely & Sometimes & Most & Always \\
\hline \multicolumn{6}{|l|}{ How often do you forget to take your medications? } \\
\hline \multicolumn{6}{|l|}{ How often do you run out of medication? } \\
\hline \multicolumn{6}{|l|}{$\begin{array}{l}\text { How often do you miss taking your medications because } \\
\text { you feel better or healthier? }\end{array}$} \\
\hline \multicolumn{6}{|l|}{$\begin{array}{l}\text { How does your health now limit you in moderate } \\
\text { activities, such as moving a table, bowling, playing golf, or } \\
\text { other activities? }\end{array}$} \\
\hline \multicolumn{6}{|l|}{$\begin{array}{l}\text { How often do you forget to get prescriptions filled at the } \\
\text { pharmacy? }\end{array}$} \\
\hline \multicolumn{6}{|l|}{$\begin{array}{l}\text { How often do you take less medication because of high } \\
\text { cost? }\end{array}$} \\
\hline $\begin{array}{l}\text { I take my medications every day at a fixed time or on fixed } \\
\text { occasions }\end{array}$ & & & & & \\
\hline
\end{tabular}

\begin{tabular}{|c|c|c|c|c|c|}
\hline & $\begin{array}{l}\text { Strongly } \\
\text { Disagree }\end{array}$ & Disagree & Neutral & Agree & $\begin{array}{l}\text { Strongly } \\
\text { Agree }\end{array}$ \\
\hline $\begin{array}{l}\text { I can mention the names of my medications, without } \\
\text { hesitation }\end{array}$ & & & & & \\
\hline $\begin{array}{l}\text { I can mention what my medications are for, without } \\
\text { hesitation }\end{array}$ & & & & & \\
\hline $\begin{array}{l}\text { I feel basically healthy. Therefore, I am sometimes unsure } \\
\text { whether I really have to take my medications daily }\end{array}$ & & & & & \\
\hline I am really afraid of the side effects of my medications & & & & & \\
\hline
\end{tabular}




\section{APPENDIX F}

Patient Satisfaction Survey

\begin{tabular}{|c|c|c|c|c|c|}
\hline & $\begin{array}{l}\text { Strongly } \\
\text { Disagree }\end{array}$ & Disagree & Neutral & Agree & $\begin{array}{c}\text { Strongly } \\
\text { Agree }\end{array}$ \\
\hline $\begin{array}{l}\text { The pharmacist provided education that will help me achieve } \\
\text { my goals of therapy }\end{array}$ & & & & & \\
\hline $\begin{array}{l}\text { The pharmacist helped me understand the intended use } \\
\text { (purpose) of my medication(s) }\end{array}$ & & & & & \\
\hline $\begin{array}{l}\text { The pharmacist helped me understand the intended results } \\
\text { (goals of therapy) of my medication(s) }\end{array}$ & & & & & \\
\hline $\begin{array}{l}\text { The pharmacist helped me to understand how to take my } \\
\text { medication(s) safely }\end{array}$ & & & & & \\
\hline I feel that I am better able to take my medication(s) correctly & & & & & \\
\hline $\begin{array}{l}\text { I feel that my overall health improved because of my visit } \\
\text { with the pharmacist }\end{array}$ & & & & & \\
\hline $\begin{array}{l}\text { I feel that my overall well-being improved because of my visit } \\
\text { with the pharmacist }\end{array}$ & & & & & \\
\hline I would recommend this service to my family and friends & & & & & \\
\hline
\end{tabular}

